\title{
Research Areas in Cloud Computing
}

\author{
Madhuri Sharma ${ }^{1}$, Parveen Kumar ${ }^{2}$ \\ ${ }^{I}$ (Department of computer science and engineering, Lovely Professional University, Phagwara, India) \\ ${ }_{2}^{2}$ (Department of computer science and engineering, Lovely Professional University, Phagwara, India)
}

\begin{abstract}
Now days every organization is migrating towards cloud computing as cloud computing is considered being more flexible and scalable as compared to other technologies. The technology simply means to provide the computing resources and services through a network. Amazon EC2, yahoo emails, Gmail, Google connect are few of the examples of cloud computing. Since cloud technology is widely adapted issues with this technology must be focused very carefully. This paper overviews the major areas which must be explored in cloud computing.
\end{abstract}

Keywords: - Cloud Computing, Cloud Gaming, Data Centers, Data Migration, Green Cloud.

\section{INTRODUCTION}

Now days when cloud computing services such as Amazon EC2, yahoo emails, Gmail, Google connect etc. becoming so popular, it seems many people are yet not clear about the word "cloud computing". Cloud computing simply means to provide the computing resources and services through a network. The provisioning of computing resources such as platform, software, hardware, database service etc is usually through internet.

According to RajKumar Buyya [2] cloud computing is defined as "a type of parallel and distributed system consisting of a collection of inter-connected and virtualized computers that are dynamically provisioned and presented as one or more unified computing resources based on service-level agreements established through negotiation between the service provider and consumers". According to Lizhe Wang [1] cloud computing defined as "a set of network enabled services, providing scalable, QoS guaranteed, normally personalized, inexpensive computing infrastructures on demand, which could be accessed in a simple and pervasive way."

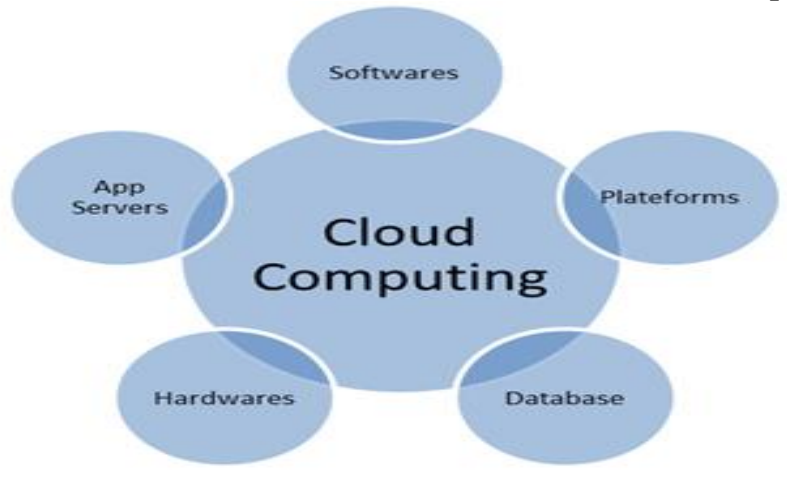

Figure 1: Cloud Computing Resources

Moreover, National Institute of Standards and Technology defines cloud computing as " a model for enabling convenient, on- demand network access to a shared pool of configurable computing resources such as networks, servers, storage, applications and services that can be frequently provisioned and released with minimal management effort or service provider interaction" [3][4]. According to NIST, this cloud model highly promotes availability as it constitutes the five important characteristics as:

\section{- On-demand:}

Cloud computing is considered to be on-demand as all the computing resources and services are provided to the end users as and when they needed without any delay. These resources released when they are no longer required and the service charged only for used resources.

\section{- $\quad$ Resource Pooling:}

Cloud computing considered to be a hub of pooled resources including certain platforms, storage, database, software and other resources. All these resources are provided to many customers from a single implementation using certain physical as well as virtual components. 


\section{- Broad Network Access:}

All the pooled resources over the clouds can be accessed via a standard network generally internet with a client side platform as endpoint (browsers).

\section{- $\quad$ Rapid Elasticity:}

Cloud computing provides its users an illusion of having infinite resource availability which they may demand any time remotely to get their work done. Moreover cloud computing considered to be highly elastic as resources can be easily pooled or removed from the data centers.

\section{- Measured Service:}

Over the cloud computing the usage of resources can be monitored, controlled and reported which provides complete transparency to both providers as well as to the consumers of the service.

Therefore we can say that cloud computing highly differs from certain traditional computing technology as cloud computing technology is considered to be highly scalable, flexible, and available that provides high level of abstraction in a way that it encapsulates as an abstract entity that provides various levels of services to its end users. That is the end users demands for a particular service and gets that service immediately without bothering how and from where this service is provided to them.

Many researchers mentioned the benefits of using cloud computing especially for business purposes. Cloud computing infrastructure consists of hardware, software, platform, databases, networks and App Servers, which allows enterprises to invest more efficiently in their hardware and software investments. So more and more enterprises are moving towards cloud computing as it helps them to reduce their computing costs via virtualization technologies. By using cloud computing the overall cost of infrastructure and administration reduced greatly moreover the enterprises also take advantage to improved utilization and faster deployments. But before heading towards cloud computing the enterprises must consider the effects, benefits as well as the drawbacks of cloud computing to the organization. It would be advisable to organizations with high level of maturity and greater technological process to use cloud computing for greater benefits.

Undoubtedly cloud computing possess many benefits, but still there are certain problems which needs to be faced and handled properly. Especially the issues of security and economics of enterprises must be researched before utilizing cloud computing. Certain issues such as The Green Cloud, Denial of Service, cloud Verification Validation and Testing, Cloud Security, Data migration, Harvesting Unused Resources and Ad hoc Cloud, Scalability in the Cloud, caching and Session State Management in the Cloud must take into account. The following sections of this paper will focus on such issues of cloud computing which must explored and handled.

\section{BENEFITS OF CLOUD COMPUTING}

Now days all the services delivered to organizations and business models are been changes tremendously by cloud computing. All the organizations prefer to adopt the outsourcing approach for infrastructures i.e. hardware, software, databases etc., rather than investing in them. Moreover cloud computing meets the requirements of flexibility, on-demand and scalability model, hence preferred by organizations so that they can upgrade and grow rapidly. Major benefits of cloud computing are as [4] [7]:

\section{- Reduced Cost:}

Cloud computing is greatly contributing to reduce the investment cost and availing the resources to organizations at very low investments. Enterprises can utilize a cloud provider, or adopting cloud paradigms internally, can save operational and maintenance expenses. All the operation can be performed by using the outsourced resources such as platforms, databases, storage space etc.

\section{- Sustainability:}

Cloud computing is considered to be highly sustainable in terms managing huge amount of resources efficiently and less consumption of energy as compared to traditional data centers. More efficiently designed clouds and data centers can face little problems or inefficiencies related to poor energy efficient clouds which consume more energy and mismanagement of service.

- Flexibility:

As in cloud computing we can easily integrate new services and resources so this technology can be considered as highly flexible. Moreover scheduling or migrations of loads can be managed and handled efficiently with clouds.

\section{- Improved Automation:}


Now days cloud computing is becoming highly automated not only in providing the services and resources, but also de-provisioned in a highly automated fashion. This improvement is ultimately increasing the efficiency of overall network in cloud computing.

\section{- Accessibility:}

Cloud computing technology is highly accessible as it makes data and services publicly available without make vulnerable sensitive information. So, users can access their data over cloud securely and remotely as and when they required.

In spite of all the above mentioned benefits of cloud computing, this technology suffers from certain issues that must be focused and handled properly.

\section{RESEARCH AREAS IN CLOUD COMPUTING}

\section{The Green Cloud:}

In cloud computing there are certain distributed resources that requires to be managed across a heterogeneous computing environment. All these resources consumes large amount of energy as they appears to be "always on" to the end users point of view. The techniques by which all the distribution of resources is done are highly inefficient in terms of energy usage. So while studying the Green Cloud computing we analyze the whole energy consumption of the computing resources, this would be based on the types of services and conditions to facilitate green cloud computing to save overall energy consumption in the related information communication systems. Now days when everything is mobile dependent we may explore green mobile communication under Green Cloud Computing. So under green cloud computing we certainly explore and research the use of virtualization in system and network resources in order to minimize energy usage while still fulfilling the service requirements and operational constraints of a cloud. Certain work has been done towards green cloud computing in context of the major causes of energy inefficiency in data centers is the idle power wasted when servers run at low utilization. The study made certainly focus on how to keep servers run at low utilization by workload consolidation. Moreover it is been claimed that the request arrival rate at servers fluctuate with time. So, in regard with these mechanisms has been developed to predict the future arrival rates from history and estimate the optimal number of servers for a class of arrival rates. Various literatures provide the solutions for dimensions such as: energy-efficient hardware, energy-aware scheduling, power-minimization in server cluster and power-minimization in mobile as well as wired networks. Feng- Seng Chu [5] focus greatly on the overall impact on energy consumption brought by cloud computing and find out when it is green.

\section{Denial of service issues in cloud computing:}

Since cloud computing dynamically provides the resources, there could the situations when the network is attacked by denial of service attacks as a result of which the cloud network, its resources and the end users keep on waiting for avail the services but won't avail it due to denial of service attacks. The attack of denial of service simply means that the users and the cloud resources are restricted to interact with each other by some third party. Resulting to this cloud may keeps on wasting the resources. So this issue must be explored and handled carefully in cloud computing. A.M. Lonea [6] made a study in which they analyze and detect the Distributed Denial of Service attacks in cloud computing environments. Such attacks provide great disruptions in the cloud network. In their study they propose a saluting to combine the evidences obtained from Intrusion Detection Systems deployed in virtual machines of the cloud systems with data fusion methodology in the frontend. So, when attacks appear, the VM-based IDS will yield alerts, which will be stored into the database of cloud. Then they provide a solution to analyze the alerts generated by IDSs using Dempster-Shafer theory operations and fault-tree analysis for the pointed attacks.

\section{Cloud VV and T Metrics:}

Verification, Validation and Testing are the basic necessities of a system evaluation and adoption but when the system and data sources are distributed such as in cloud computing all these activities are performed in random manner. The general testing strategies and models for testing the code, application and the design may not be applicable in a cloud or may be the software developed for centralized environment may not work in the same way with cloud computing. There are certain areas of research in validation, verification and testing of cloud from formal verification to empirical research and metric validation of multipart or parallel analysis. In cloud computing Testing can be applied to systems, security, architecture models and other constructs within the cloud environment. Apart from this failure analysis, taxonomies, error handling and recognition are all related areas of research under cloud verification, validation and testing in cloud computing. Jarry Gao [15] made a study and provides a comprehensive tutorial on cloud testing and cloud-based application testing. This paper provides a complete overview about cloud testing and thoughts in minds of engineers and managers in context of it. This paper provides a clear vision for web based software testing and cloud based software applications. In 
this paper they also highlight and compare different commercial products and solutions that supports cloud testing.

\section{Cloud Security:}

Cloud security is the major concern when we talk about distributed networks and cloud computing. All the clouds, data centers, stored applications and other architectures must be protected with appropriate and dependable securities. Certain issues must be taken into account while providing cloud security such as: should security be centralized or decentralized? Who should manage the security services? Type of security required? Hassan Zaki [8] introduces four cloud security problems as: XML Signature Element Wrapping, Browser Security, Cloud Malware Injection Attack and Flooding Attacks and proposes the counter measures. Hongwei Li [9] focus on authentication over the cloud, both of users and services in a significant issue for the trust and security of the cloud computing. The paper is based on identity-based hierarchical model for cloud computing and certain encryption and signature schemes and presents an identity-based authentication protocol for cloud computing and services.

\section{Data Migration:}

In distributed networks and cloud computing the cost and time required to move data over the network is a major issue. Certain issues such as: how to migrate data from one location to another over the cloud, where to store that data, how to provide data to its users, load balancing and job scheduling over the cloud in various data centers must be focused under data migration in the cloud. The aim of this study is to investigate the issues that may affect data migration in the cloud and to propose mechanisms to specify policies for data migrations. Samuil Islam [11] proposed a design which hosts various data centers in certain remote locations where each data centre may hold multiple databases on a pool of servers. Salim Bitam [10] made a study to resolve the issue of job scheduling in various data centers over the cloud. An algorithm is also proposed by him for efficient job scheduling based on bees life.

\section{Harvesting unused resources and Ad hoc Cloud:}

In cloud computing there might be certain situations when ad-hoc cloud is required to provide cloud services, rather than introducing dedicated machines within the data centers, that the existing infrastructure of the organization is not fulfilling. In case a hug number of ad-hoc resources are placed over cloud even if they are of no longer use, the overall performance of cloud network decreases. So the goal of this study is to investigate which are the ad-hoc computing resources within an enterprise, how the underused computing resources within an enterprise may be harvested and harnessed to improve return on IT investment. The study made for this will make all the harvested resources available in a form of ad-hoc clouds, the architectures and deployment models of which depends on the supply of resources and demands for cloud services. Alaa Eldeen S Ahmed [12] made a study to manage and utilize cloud computing underused resources. In this study they propose a new approach to model an efficient and improved IaaS services. This improved approach focus on extending the available cloud computing platform infrastructure by harvesting underused genetic computing resources that are available broadly in public domains. The proposed approach aims to improve the computing power for the cloud computing platform without charging any extra cost.

\section{Scalability in the Cloud:}

The cloud so deployed must be highly scalable. High scale cloud can be achieved by two ways: Scale up i.e. getting bigger VM or scale out i.e. more VMs. Certain companies provide high scaled clouds this way eg. Amazon EC2, Azure, Google App Engine. Aarti Singh [16] made a study to ensure reliable, secure, sustainable and scalable infrastructures in cloud computing for hosting internet based application services. In their study they propose an agent based framework for providing scalability in cloud computing environment which is supported by an algorithms for evaluating another cloud when the approachable cloud becomes overloaded and for searching closest datacenters with least response time of virtual machine.

\section{Session State Management in Cloud:}

The session state is used to store and retrieve information or values for a user across ASP.Net pages in a web application. There are four available modes to store session values in ASP.NET: State Server which stores session state in another process called ASP.NET state service, SQL Server which stores session state in a SQL Server database, In-Proc which stores session state in the individual web server's memory, and Custom which lets you choose a custom storage provider. So, this study focuses greatly on how session state is managed and implemented in cloud for web applications. Example in Azure standard session state management for ASP.NET applications is done in memory, which will not work where an application is scaled out over multiple instances. In an Alternative session state management involving storing the state in persistent store need to be used instead 
say Azure tables or SQL Azure. So we may compare and contrast various session state management techniques and their features on different cloud platforms such as Azure, Google App Engine etc.

\section{Cloud Gaming:}

Now days when cloud computing is expanding so rapidly, on-demand gaming known as cloud gaming is also most popular application of cloud these days. A lot of work has been done to make this application more efficient and convenient for its end users. Gwendal Simon [14] made a study in this context. They demonstrate via a large-scale measurement study that the current cloud computing infrastructure is not able to meet the requirements necessary for acceptable game play for hug number for end users, which is limiting the number of users for an on-demand gaming service. Furthermore Chun- Ying Huang [13] made a study towards cloud gaming and propose a cloud gaming system named as Gaming Any Where, which is considered as a first open cloud gaming system. The proposed is also considered to be highly extensible, portable and reconfigurable.

\section{Caching in Cloud:}

Under this area of research an investigation for caching solutions for web applications in clouds is done. In this we need to examine all types of caching that the cloud provides such as CDN in Azure. Here we also need to focus on output of caching and the caching of application data example asp.net caching in Azure, distributed caching using memcached or windows Azure AppFabric Caching. We may compare and contrast various caching solution and examine performance improvements that can be obtained from use of these technologies.

\section{CONCLUSION}

Since Cloud Computing is expanding rapidly and is widely adapted, all the areas must be investigated very carefully. On the bases of investigations and information gathered certain measures must be taken to improve the overall performance, security, reliability and availability of the system. The major research areas have been discussed in this paper over which certain investigations can be made, but there may be yet other domains under cloud computing which must be focused and investigated.

\section{ACKNOWLEDGMENTS}

\section{I, Madhuri Sharma}

would like to express my gratitude to all those who gave me the possibility to complete this work. I would like to thank my GOD for giving strength to complete this task and to my mentor,

\section{Mr. Parveen Kumar}

for providing support and material related to the area of this research. His valuable suggestions also helped me to stay focused and follow the path described by him.

I would also like to thank to my department heads, management of LPU for supporting me throughout the entire work and contributing to the completion of this dissertation work in their own distinct way.

\section{REFERENCES}

[1] Lizhe Wang, Gregor von Laszewski, Andrew Younge, Xi He, Marcel Kunze, Jie Tao, and Cheng Fu (2008) "Cloud Computing: a Perspective Study." New Generation Computing 28, no. 2 pp: 137-146.

[2] Wickremasinghe, Bhathiya, and Rajkumar Buyya. (2009) "CloudAnalyst: A CloudSim-based Tool for Modelling and Analysis of Large Scale Cloud Computing Environments." MEDC Project Report 22.6

[3] Sriram, Ilango, and Ali Khajeh-Hosseini (2010) "Research agenda in cloud technologies." arXiv preprint arXiv: 1001.3259 .

[4] Kapil Bakshi, (2011) "Considerations for cloud data centers: Framework, architecture and adoption." Aerospace Conference, IEEE. Version 2, pp 1-7

[5] Feng-Seng Chu, Kwang-Cheng Chen and Chen-Mou Cheng "Toward Green Cloud Computing" ACM, February, 2011

[6] A.M.Lonea, D.E.Popescu and H.Tianfield "Detecting DDoS Attacks in Cloud Computing Environment", ISSN Frbruary, 2013 pp-70-78

[7] Rao, N. Mallikharjuna, C. Sasidhar, and V. Sathyendra Kumar. (2010) "Cloud computing through mobile-learning." (IJACSA) International Journal of Advanced Computer Science and Applications, Vol. 1, no. 6, pp: 42-47.

[8] Hassan Zaki and Danish Jamil "Security Issues in Cloud Computing and Counter Measures", April,2011, Vol.3 pp 2672-2676

[9] Hongwei Li, Yuanshun Dai, Ling Tian and Haomiao Yang "Identity- Based Authentication for Cloud Computing" ,2009 LNCS 5931,pp 157-166 
[10] Salim Bitam. (2012) "Bees Life Algorithm for job scheduling in cloud computing." International Conference on Computing and Information Technology. ICCIT, pp 186- 191

[11] Samil Islam and Farah Habib Chanchary "Data Maigration: Connecting Databases in the Cloud" ICCIT 2012, pp: 450-455

[12] Alaa Eldeen S Ahmed, Abdulwahab K Alsammak and Essam Algizawy. Article: A New Approach to Manage and Utilize Cloud Computing Underused Resources. International Journal of Computer Applications 76(11):29-36, 2013

[13] Huang, Chun-Ying, "GamingAnywhere: An open cloud gaming system." Proceedings of the 4th ACM Multimedia Systems Conference. ACM, 2013.

[14] Gwendal Simon, Catherine Rosenbery, Sharon Choy and Bernard Wong "The Brewing Storm in Cloud Gaming: A Measurement Study on Cloud to End-User Latency" IEEE, 2012.

[15] Jerry Gao, Xiaoying Bai and Wei-Tek Tsai "Cloud Testing- Issues, Challenges, Need and Practice" SEIJ Vol. 1, No. 1, 2011 pp: 9-23

[16] Aarti Singh, Manisha Malhotra, "Agent Based Framework for Scalability in Cloud Computing", ISSN 2229-3345 Vol. 3, No. 4, 2012, pp: 41-45 